\title{
Consumo de antiinflamatorios no esteroideos en pacientes que concurren por primera vez a un Servicio de Reumatología
}

\author{
M.J. Santa Cruz, M.A. Medina, S.B. Papasidero, R. Chaparro del Moral, M. Guinsburg, J.A. Caracciolo, O.L. Rillo \\ Servicio de Reumatología, Hospital General de Agudos Dr. E. Tornú (CABA).
}

\begin{abstract}
Resumen
Introducción: El uso de antiinflamatorios no esteroideos (AINES) conlleva una mayor probabilidad de enfermedad gastrointestinal y cardiovascular en pacientes con factores de riesgo. Por tal motivo, se desarrollaron diversas recomendaciones con el fin de prevenir dichas complicaciones.

Objetivo: Evaluar la frecuencia del consumo de AINES al momento de consultar por primera vez a un Servicio de Reumatología de demanda espontánea; y analizar tanto la conducta del médico así como el correcto cumplimiento de las recomendaciones para la toma de estos fármacos al finalizar la consulta.

Material y métodos: Estudio observacional de corte transversal.

Resultados: Se incluyeron 304 pacientes, 59,5\% consumían AlNES. La mayoría de estos pacientes presentaban riesgo gastrointestinal moderado/alto, sólo 28,3\% recibían adecuada protección. De aquellos pacientes que recibían AINES, se consideró que requerían dicho tratamiento el 50\%. Al finalizar la consulta, se tomó una conducta correcta en el $89 \%$ de los casos en relación al riesgo gastrointestinal.

Conclusión: Más de la mitad de los pacientes consumían AINES. Se observó un buen cumplimiento de las recomendaciones por los médicos tratantes al finalizar la primera consulta. Sin embargo, es importante mencionar que el uso indiscriminado de AINES tanto por parte de los pacientes como por parte de los médicos sigue siendo alto.
\end{abstract}

Palabras clave: antiinflamatorios no esteroideos, riesgo gastrointestinal, riesgo cardiovascular, recomendaciones, drogas gastroprotectoras.

\section{Abstract}

Introduction: The use of Non-Steroidal Anti-Inflammatory Drugs NSAID (NSAIDS) leads to a higher probability of gastrointestinal and cardiovascular disease in patients with risk factors. Therefore, a number of recommendations were developed to prevent these complications. The aim of this study was to evaluate the intake frequency of NSAID by patients who attend to a rheumatology department for the first time; and to analyze the attending physicians' prescription as well as the correct implementation of the recommendations for the use of these drugs once this first visit was ended. Methods: We perform a cross-sectional observational study. Results: Three hundred and four patients were included, 59.5\% were taking NSAIDs. Most of these patients had moderate/high gastrointestinal risk and only $28.3 \%$ received adequate protection. Of patients receiving NSAIDs, it was considered that only $50 \%$ required such treatment. At the end of the first visit, the attending physicians made the correct implementation of the recommendations in $89 \%$ of cases in regard to gastrointestinal risk.

Conclusion: More than half of the patients were taking NSAIDS. A good compliance with the recommendations by the attending physicians at the end of the first visit was observed. However, it is noteworthy that the indiscriminate use of NSAIDs, both by patients and by physicians, remains high.

Key words: non-steroidal anti-inflammatory drugs, gastrointestinal risk, cardiovascular risk, recomendations, gastroprotective agents. 


\section{Introducción}

Los antiinflamatorios no esteroideos (AINES) son fármacos ampliamente utilizados en el ámbito de la Reumatología, habiendo sido bien documentada su eficacia para el dolor y la inflamación ${ }^{1}$. Se estima que más de 30 millones de personas en todo el mundo consumen AINES diariamente para el control de dichos síntomas ${ }^{2}$. Debido a las marcadas diferencias que estos fármacos presentan en su perfil de seguridad y siendo conocido el impacto que los mismos tienen tanto sobre el aparato gastrointestinal como cardiovascular, en abril de 2005 la FDA (Food and Drugs Administration) determinó que todos los AINES debían llevar una etiqueta de advertencia resaltando el potencial riesgo aumentado de eventos cardiovasculares (CV) serios y gastrointestinales (GI) con riesgo de vida ${ }^{3}$. Además, por el aumento en la probabilidad de enfermedad gastrointestinal y cardiovascular en pacientes con factores de riesgo que consumen AINES, distintas sociedades médicas tanto de Europa como de América han desarrollado diversas recomendaciones con el fin de prevenir dichas complicaciones. Por lo tanto, los objetivos de este trabajo fueron evaluar la frecuencia del consumo de AINES al momento de consultar por primera vez a un Servicio de Reumatología de demanda espontánea; y analizar tanto la conducta del médico así como el correcto cumplimiento de las recomendaciones para la toma de estos fármacos al finalizar la consulta.

\section{Materiales y métodos}

Se realizó un estudio observacional de corte transversal en el cual se incluyeron pacientes consecutivos mayores de 18 años que concurrieron por primera vez al consultorio de demanda espontánea de nuestro servicio durante el período comprendido entre el 2 de enero al 30 de mayo de 2014 por padecer dolor musculo-esquelético. Se detallaron por interrogatorio características demográficas, motivo de consulta, comorbilidades, tabaquismo, tratamiento actual [consumo de AINES (dosis, modo de uso reglado o intermitente e indicación médica), uso de Aspirina a bajas dosis, Inhibidores de la Bomba de Protones (IBP), Ranitidina, Esteroides, Anticoagulantes orales (ACO)]. Se consideró dosis supra-óptima aquella que supera la dosis máxima recomendada. Al examen físico, se consignó tensión arterial (TA), peso, talla y se calculó el Índice de Masa Corporal (IMC). Además se registró el perfil lipídico en aquellos pacientes de quienes se disponía dicha información. Los pacientes fueron divididos en dos grupos (AINES y NO AINES) según el tratamiento que recibían al momento de la consulta. También se consignó el tratamiento indicado al finalizar la consulta. Para realizar el cálculo de riesgo GI se tuvo en cuenta el Consenso de la Sociedad Española de Reumatología y del Colegio Mexicano de Reumatología para el uso apropiado de AINES. Según éste, se consideran como factores de riesgo GI: el antecedente de úlcera gastroduodenal (UGD) complicada o no complicada, el uso de ACO, edad $>65$ años, el uso concomitante de más de un AINE (incluyendo Aspirina a bajas dosis), tratamiento con dosis altas de AINES y duración prolongada del mismo. Se consideran pacientes con alto riesgo GI aquellos con antecedente de UGD complicada o que utilizan ACO o que presenten más de dos factores de riesgo; pacientes con moderado riesgo GI aquellos que presenten un factor de riesgo (que no incluya ninguno de los mencionados previamente) y bajo riesgo GI aquellos sin factores de riesgo ${ }^{4}$. Es importante mencionar la indicación en dicho Consenso de utilizar IBP en aquellos pacientes que refieren antecedente de dispepsia relacionada con el uso de AINES. Si bien el uso de esteroides no es considerado en este consenso como un factor de riesgo GI, debido al amplio uso de los mismos en nuestro ámbito y a la importante evidencia además de otras guías y recomendaciones que los considera como tal ${ }^{5,6,7,8}$, es que decidimos incluirlos como otro factor de riesgo a tener en cuenta. Con respecto al riesgo $\mathrm{CV}$, se emplearon las tablas de predicción para el mismo desarrollada para América Latina de la Organización Mundial de la Salud (OMS) y la Sociedad Internacional de Hipertensión. En dichas tablas, se evalúan las siguientes variables: el sexo, edad, presión arterial sistólica, colesterol total en sangre, consumo de tabaco y la presencia o ausencia de diabetes mellitus. La combinación de las mismas permite la categorización de los pacientes en aquellos que presentan riesgo $<10 \%$, entre $10 \%-<20 \%$, entre $20 \%-<30 \%$, entre $30 \%-<40 \%$, y riesgo $\mathrm{CV} \geq 40 \%$ de padecer un episodio cardiovascular, mortal o no, en un período de 10 años 9 . Análisis de los datos: Se realizó estadística descriptiva de las variables estudiadas. La comparación por grupos (AINES vs. NO AINES) se realizó mediante Test de $\mathrm{Chi}^{2}$ o prueba exacta de Fisher para variables categóricas y mediante prueba $t$ o pruebas no paramétricas según cada caso para las variables continuas.

\section{Resultados}

Se incluyeron 304 pacientes (81\% mujeres), con un promedio de edad de 56 (DE 15) años. El mayor número de pacientes se encontró en el grupo etario de 51 a 70 años $(41,8 \%)$, seguido por 50 pacientes $(16,4 \%)$ de 41 a 50 años, 
y luego por 30 pacientes $(9,9 \%)$ de 71 a 80 años. Las causas más frecuentes de consulta fueron: poliartralgias $(57,6 \%)$ y gonalgia $(10,2 \%)$; seguidas de dolor en manos $(8,9 \%)$, lumbalgia $(7,6 \%)$, dolor en pies $(2,3 \%)$, cervicalgia $(1,6 \%)$, dolor en cadera y tobillo (1\%), y dolor en codos $(0,3 \%)$. Cinco por ciento de los pacientes consultaron por otros motivos (dolor de características neuropáticas en miembros superiores y/o inferiores, dolor generalizado, dolor muscular, etc.) (Ver Gráfico 1).

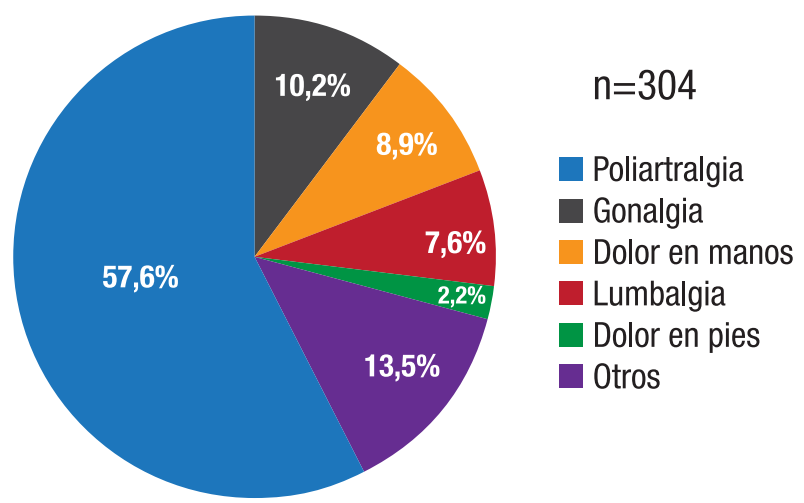

Gráfico 1. Motivos de consulta.

Las comorbilidades más prevalentes fueron diabetes (7,6\%) y accidente cerebrovascular (4,6\%). El 16,8\% eran tabaquistas. Sólo 2 pacientes $(0,7 \%)$ presentaban diabetes con compromiso renal, y $2(0,7 \%)$ tenían antecedente de infarto agudo de miocardio. Con respecto a los antecedentes y síntomas GI, 39,5\% (120/304) referían dispepsia;

\begin{tabular}{|l|c|c|}
\hline & Número de Pacientes (n) & Porcentaje (\%) \\
\hline Mujeres & 247 & $81,25 \%$ \\
\hline Hombres & 57 & $18,75 \%$ \\
\hline Edad, media (DE) & \multicolumn{2}{|c|}{$56,14(14,7)$} \\
\hline Tensión Arterial, media (DE) & $130(22,24) / 81(13,4)$ \\
\hline IMC, media (DE) & \multicolumn{2}{|c|}{$27,9(5,8)$} \\
\hline Dispepsia & 120 & $39,5 \%$ \\
\hline Tabaquismo & 51 & $16,8 \%$ \\
\hline DBT & 23 & $7,6 \%$ \\
\hline ACV & $14 \%$ & $4,6 \%$ \\
\hline Esteroides & $14 \%$ & $4,6 \%$ \\
\hline Hemorragia digestiva & 3 & $1 \%$ \\
\hline UGD & 3 & $1 \%$ \\
\hline
\end{tabular}

P= NS, IMC: Índice de masa corporal, DBT: Diabetes mellitus, ACV: Accidente cerebrovascular, UGD: Úlcera gastroduodenal.

Tabla 1. Datos generales de la población del estudio. Número total de pacientes $(n)=304$. el 1\% (3/304) antecedente de hemorragia digestiva, y la misma cantidad de pacientes antecedente de UGD (Ver Tabla 1).

Los valores de TA promedios fueron: 130 (DE 22)/81 (DE 13) $\mathrm{mmHg}$; IMC promedio: 27,9 (DE 6) $\mathrm{kg} / \mathrm{m}^{2}$. En cuanto al consumo de AINES: 168 pacientes recibía sólo un $\operatorname{AINE}(55,3 \%), 13(4,2 \%)$ más de un AINE, y 123 $(40,5 \%)$ no recibían AINES al momento de la consulta. No se hallaron diferencias significativas en las variables evaluadas entre los pacientes que utilizaban AINES y aquellos que no lo hacían (Ver Gráfico 2).

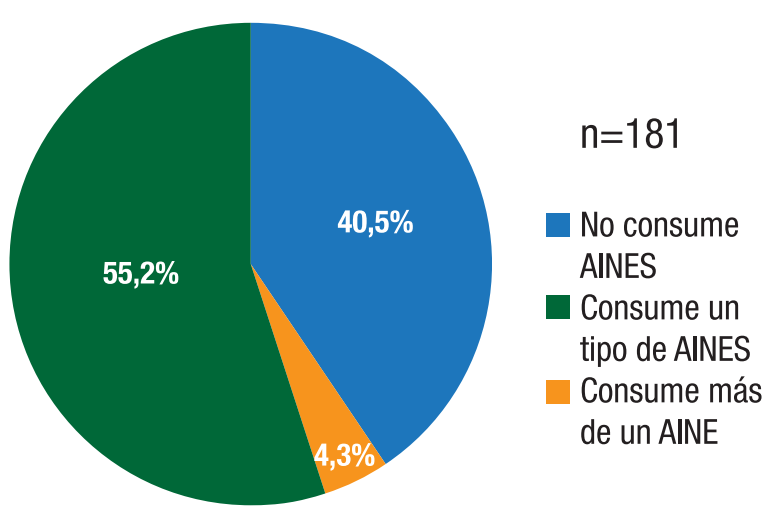

Gráfico 2. Consumo de AINES.

De los pacientes que consumían AINES ( $\mathrm{n}=181$ ), el $53 \%$ lo hacían en forma reglada; sólo un 4,3\% recibía dosis supra-óptimas; los más utilizados fueron Ibuprofeno $(42,3 \%)$ y Diclofenac $(39,6 \%)$, seguidos por Meloxicam $(11 \%)$ y Aspirina $(2,75 \%)$; sólo un paciente empleaba un inhibidor específico de la COX2 (Ver Gráfico 3).

El $42 \%$ de los pacientes recibían AINES por prescripción médica, de los cuales en el 38,2\% de los casos la

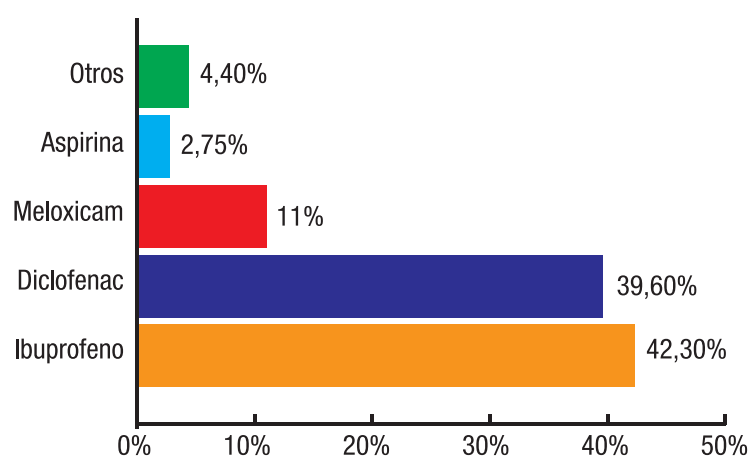

Gráfico 3. Tipos de AINES utilizados. 
indicación fue realizada por parte del traumatólogo, 29\% por su médico de cabecera, $14,5 \%$ por un reumatólogo, $10,5 \%$ en la guardia externa y en un menor porcentaje por otras especialidades (Ver Gráfico 4).

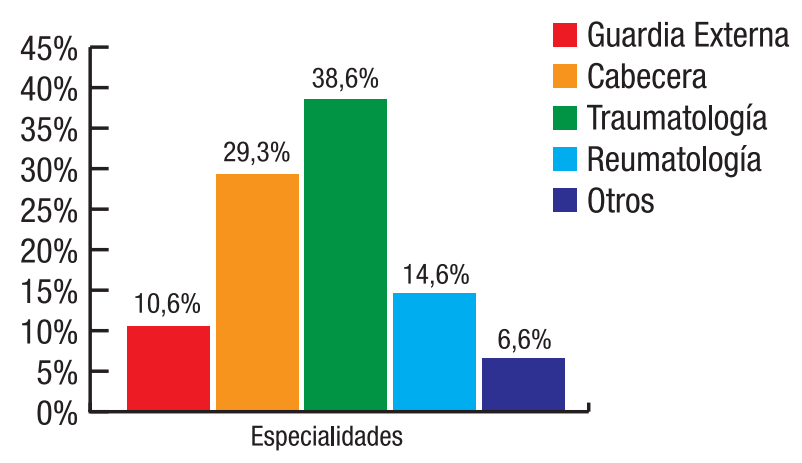

Gráfico 4. Indicación de AINES por especialidad.

De los 181 pacientes que referían consumir algún tipo de AINE previo a la consulta, 40,9\% (74/181) presentaban bajo riesgo GI, 36,5\% (66/181) riesgo GI moderado y 22,6\% (41/181) riesgo GI alto (Ver Gráfico 5).

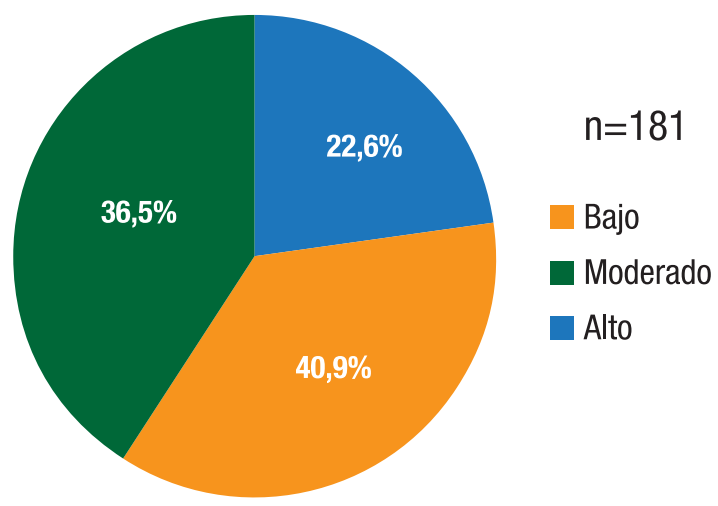

Gráfico 5. Riesgo gastrointestinal en los pacientes consumidores de AINES.

De estos dos últimos grupos, $71,7 \%$ (76 pacientes) no utilizaban protección gástrica. Con respecto al tratamiento con IBP en los pacientes que ingerían AINES, pudimos observar que de aquellos que presentaban riesgo GI moderado ( $\mathrm{n}=66$ ), 80,3\% no consumían IBP; y de los que pertenecían al grupo con riesgo GI alto $(n=41)$, el $61 \%$ no utilizaban ningún tipo de fármaco gastroprotector (Ver Tabla 2).

Consideramos importante mencionar que el $63 \%$ de aquellos pacientes que recibieron AINES por indicación médica antes de consultar a nuestro servicio, no presenta-

\begin{tabular}{|l|c|c|}
\hline \multicolumn{1}{|c|}{$\mathbf{n = 1 8 1}$} & Con IBP $\mathbf{n = 3 6}$ & Sin IBP $\mathbf{n = 1 4 5}$ \\
\hline Riesgo Gl bajo $\mathrm{n}=74$ & $7(9,5 \%)$ & $67(90,5 \%)$ \\
\hline Riesgo Gl moderado $\mathrm{n}=66$ & $13(19,7 \%)$ & $53(80,3 \%)$ \\
\hline Riesgo Gl alto $\mathrm{n}=41$ & $16(39 \%)$ & $25(61 \%)$ \\
\hline
\end{tabular}

Tabla 2. Consumo de Inhibidores de la Bomba de Protones (IBP) en pacientes consumidores de AINES.

ban la protección gástrica adecuada según lo recomendado por los consensos mencionados. Sólo 2,8\% (5 pacientes) consumían Ranitidina, la cual no ha demostrado ser útil en dosis habituales para la protección gástrica en aquellos pacientes que reciben AINES. La misma era utilizada en dosis sub-óptimas.

De los 181 pacientes que consumían AINES previo a la consulta, el 50\% continuaron con dicha indicación luego de ser evaluados por los médicos de nuestro centro; $y$ con respecto a aquellos pacientes que no los recibían, se indicaron los mismos en el 31,7\%. Dentro de los fármacos prescriptos, el 41,3\% recibieron Meloxicam, 25,4\% Ibuprofeno, $16,7 \%$ Diclofenac, $15 \%$ Naproxeno, $1,6 \%$ otro tipo de AINE. El médico tratante al final de la consulta tomó una conducta correcta según las recomendaciones en el 89\% de los casos en relación a la indicación de AINES e IBP con respecto al riesgo GI. Dentro de los motivos por los cuales se consideró que la conducta tomada era errónea, se evidenció que no se indicó IBP en 7 pacientes que presentaban varios factores de riesgo GI asociados y en otros 7 que referían el antecedente de dispepsia; por otro lado, en 14 sujetos con bajo riesgo GI se indicó un IBP.

Se evaluó también el riesgo CV en 270 pacientes: $85 \%$ presentaban un riesgo $<10 \%$, en el $11 \%$ el riesgo se ubicó entre $10 \%-<20 \%$, en el $2 \%$ entre $20 \%-<30 \%, 1,5 \%$ entre $30 \%-<40 \%$, y sólo $0,3 \%$ presentó un riesgo $\mathrm{CV} \geq 40 \%$ (Ver Gráfico 6).

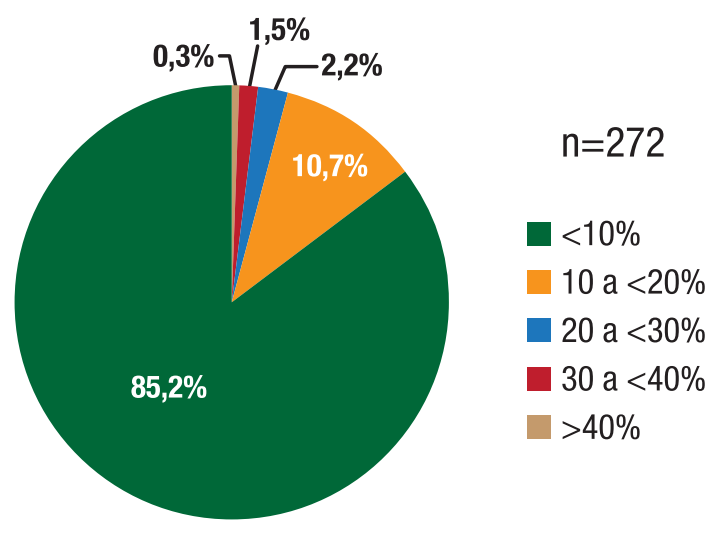

Gráfico 6. Riesgo cardiovascular. 
Con respecto al grupo de riesgo CV y el tipo de AINE indicado, pudimos observar que en aquellos pacientes con riesgo leve, el AINE más frecuentemente indicado fue el Meloxicam, siendo el Ibuprofeno junto con el Meloxicam igualmente utilizados en los grupos de riesgo moderado y alto. Con respecto a los grupos con riesgo CV moderado/alto, en ningún caso se indicó inhibidores específicos de la COX-2. No hallamos diferencias significativas en el riesgo $\mathrm{CV}$ entre los pacientes consumidores de AINES y aquellos que no los recibían (Ver Tabla 3).

\begin{tabular}{|c|c|c|c|c|}
\hline Riesgo CV & \multicolumn{2}{|c|}{ Con AINES } & $(\mathbf{n = 1 6 2})$ & \multicolumn{2}{c|}{ Sin AINES } & $(\mathbf{n = 1 1 0})$ \\
\hline & $\mathrm{n}$ & $\%$ & $\mathrm{n}$ & $\%$ \\
\hline$<10 \%$ & 138 & $85,2 \%$ & 94 & $85,5 \%$ \\
\hline $10 \mathrm{a}<20 \%$ & 16 & $9,9 \%$ & 13 & $11,8 \%$ \\
\hline $20 \mathrm{a}<30 \%$ & 6 & $3,7 \%$ & 0 & 0 \\
\hline $30 \mathrm{a}<40 \%$ & 2 & $1,23 \%$ & 2 & $1,8 \%$ \\
\hline$>40 \%$ & 0 & 0 & 1 & $0,9 \%$ \\
\hline
\end{tabular}

Tabla 3. Consumo de AINES según riesgo CV ( $n=272)$.

\section{Discusión}

En el presente estudio, evaluamos el uso de AINES en pacientes que concurrían por primera vez por dolor al consultorio de demanda espontánea de un Servicio de Reumatología durante un período de 5 meses; así como el cumplimiento de las recomendaciones con respecto a la protección del riesgo GI y CV. Pudimos constatar que el 59,5\% de los pacientes que consultaron consumían AINES previo a la consulta. Al finalizar la misma, se decidió indicar dicho tratamiento al 50\% de los pacientes. En un estudio retrospectivo realizado en el 2013 en nuestro servicio, observamos resultados similares al presente trabajo ${ }^{10}$. Un porcentaje mayor fue reportado por Lanas y cols. en un estudio prospectivo que incluyó 1040 pacientes que padecían dolor, en el cual se indicaron AINES en el 74\% de los casos $^{11}$. Sin embargo, en otro estudio retrospectivo de Koffeman y cols. donde se evaluó el riesgo CV y el uso de AINES, se evidenció que sólo al 25\% de los pacientes que consultaron por dolor musculo-esquelético se les indicó un $\mathrm{AINE}^{12}$, presentando una marcada diferencia con respecto a los trabajos mencionados anteriormente.

Con respecto al riesgo GI hallado en nuestra población, pudimos observar que el $60 \%$ de los pacientes presentaba riesgo GI moderado/alto. Estos resultados difieren con los observados en los Países Bajos, donde Sturkenboom y cols., en un estudio retrospectivo que incluyó 69.648 pacientes, concluyen que el $22 \%$ presentaban riesgo GI moderado/alto ${ }^{13}$. Valores similares a este último (29\%) fueron reportados por Koffeman y cols. en una cohorte de 474.201 pacientes incluidos a lo largo de 10 años ${ }^{12}$. Esta discrepancia con nuestro trabajo podría atribuirse a la variación en el tamaño muestral, como así también entre otros a la etnia, diferencias socioculturales, etc.

En relación al cumplimiento de las guías y recomendaciones para el uso de AINES, lo que observamos fue que en el $89 \%$ de los casos el médico tratante tomó una conducta acertada en función a los factores de riesgo GI del paciente. Sin embargo, no podemos dejar de mencionar que si consideramos aquellos pacientes que habían recibido la prescripción de algún AINE previo a la consulta a nuestro servicio, los datos son menos favorables, pues sólo en el $63 \%$ de los casos el médico había tomado una conducta preventiva con respecto al uso de estos fármacos. Hemos encontrado datos menos alentadores en la bibliografía, como en el trabajo de Sturkenboom y cols. mencionados previamente, donde se evidencia que hasta el $81 \%$ de los pacientes que presentaban dos o más factores de riesgo GI según las guías del Colegio Americano de Reumatología, no recibían adecuada protección ${ }^{13}$. En otro estudio del grupo de van Dijk, objetivaron que sólo el 23\% de los pacientes que consumían AINES recibían algún tipo de medida preventiva ${ }^{14}$. En varios estudios realizados en Estados Unidos, Alemania, Canadá y Francia entre otros, la proporción de pacientes que consumen AINES con IBP en forma adecuada es muy baja, ubicándose entre el 25 al 50\%,15-22. Es de destacar que en los Países Bajos el conocimiento y difusión de mejores estrategias gastroprotectoras ha modificado la prevalencia de su aplicación, aumentando del 5,1\% al 15,9\% entre el período de 1996 al 2002 ${ }^{1}$.

Finalmente, el riesgo CV en nuestra cohorte era bajo en más del $85 \%$ de los pacientes, siendo de relevancia mencionar que la edad media fue de 56 (DE 15) años. En la cohorte del grupo de Sturkenboom hallaron datos similares, presentando el $77 \%$ de la población riesgo $\mathrm{CV}$ bajo. En dicho estudio, en los pacientes que presentaban alto riesgo, se indicó AINES tradicionales en el 19\% e inhibidores COX-2 específicos en el 2,2\% de los casos, evidenciando la menor probabilidad de prescribir estos últimos en quienes presentaban riesgo CV elevado ${ }^{13}$. En nuestro trabajo no se pudo evaluar el cumplimiento de las recomendaciones en relación al uso de AINES y riesgo $\mathrm{CV}$ debido al escaso número de pacientes con riesgo $\mathrm{CV}$ alto.

Como fortalezas de nuestro estudio, podemos mencionar el diseño observacional de tipo transversal el cual nos permitió una recolección más exacta de los datos a re- 
cabar. Además, por tratarse de un consultorio de demanda espontánea, se detalló el consumo de AINES no sólo en pacientes evaluados previamente por otro profesional de la salud sino que también pudimos evaluar la frecuencia de automedicación en nuestra población. Dentro de las limitaciones, en primer lugar debemos mencionar el bajo número de pacientes en relación a otras cohortes. En segundo lugar, no fue posible evaluar el motivo por el cual el médico tratante realizó la indicación de la estrategia gastroprotectora, lo cual podría llevar a una sobreestimación del cumplimiento de la guía. Por último, en nuestra población en particular no fue posible evaluar el cumplimiento de las recomendaciones existentes para el uso de AINES en relación al riesgo $\mathrm{CV}$, ya que sería necesario un mayor porcentaje de pacientes con riesgo CV elevado.

En conclusión, en nuestro estudio hemos observado que más de la mitad de los pacientes que padecían dolor, consumían AINES en forma habitual. Pudimos evidenciar que la mayoría presentaban riesgo GI moderado/alto, con un bajo porcentaje de los mismos recibiendo una adecuada protección GI. Con respecto a la conducta tomada por el médico tratante, se consideró que de aquellos que recibían AINES previo a esta primera consulta, la mitad requerían continuar con dicho tratamiento. Finalmente, a diferencia de estudios similares, pudimos observar un buen cumplimiento de las recomendaciones por los médicos tratantes al finalizar la primera consulta. Sin embargo, es importante mencionar que el uso indiscriminado de AINES tanto por parte de los pacientes como por parte de los médicos sigue siendo alto. Con el objetivo de reducir las complicaciones asociadas con el empleo de estos fármacos, consideramos imprescindibles una amplia y correcta difusión y aplicación de las guías y recomendaciones para la prevención de sus posibles efectos lesivos.

\section{Bibliografía}

1. Sturkenboom MCJM, Burke TA, Dieleman JP, Tangelder MJD, Lee F. Underutilization of preventive strategies in patients receiving NSAIDs. Rheumatology (Oxford).2003 Nov; 42 Suppl 3:iii23-31.

2. Zhang J, Ding E.L, Song Y. Adverse effects of cyclooxygenase 2 inhibitors on renal and arrhythmia events: meta-analysis of randomized trials. JAMA. 2006; 296:1619-32.

3. US Food and Drug Administration: Drug Information.COX-2 selective. Accessed August 8, 2006

4. Segura GB, Hernández Cruz B, Gobboc M, Lanas
Arbeload A, Salazar Paramoe M. Uso apropiado de los antiinflamatorios no esteroideos en reumatología: documento de consenso de la Sociedad Española de Reumatología y el Colegio Mexicano de Reumatología. Reumatol Clin. 2009; 5(1):3-12.

5. Burmester G, Lanas A, Biasucci L, Hermann M, Lohmander S, Olivieri I. The appropriate use of non-steroidal antiinflammatory drugs in rheumatic disease: opinions of a multidisciplinary European expert panel. Ann Rheum Dis 2011; 70:818-22.

6. Dubois RW, Melmed GY, Henning JM, Laine L. Guidelines for the appropriate use of non-steroidal anti-inflammatory drugs, cyclo-oxygenase-2-specific inhibitors and proton pump inhibitors in patients requiring chronic anti-inflammatory therapy. Aliment Pharmacol Ther 2004; 19:197-208.

7. Chan FKL, Graham DY. Review article: prevention of non-steroidal anti-inflammatory drug gastrointestinal complications - review and recommendations based on risk assessment. Aliment Pharmacol Ther 2004; 19:1051-61.

8. Lanza FL, Chan FKL, Quigley EMM. Guidelines for Prevention of NSAID-Related Ulcer Complications. Am J Gastroenterol 2009; 104:728-38.

9. Tabla de predicción de riesgo AMR B de la OMS/ ISH. Guía de bolsillo para la estimación y el manejo del riesgo cardiovascular: Prevención de las Enfermedades Cardiovasculares. Guías del Ministerio de Salud (Junio 2009).

10. Guinsburg M, Santa Cruz MJ, Papasidero SB, Rillo OL. Uso inadecuado de AINES en pacientes que concurren por primera vez a la consulta en un Servicio de Reumatología. [abstract]. Rev Arg Reumatol 2013; 24 (Supl 5):56.

11. Lanas A, Polo Tomas M, Roncales P, Gonzalez M, Zapardiel J: "Prescription of and Adherence to Non-steroidal Antiinflammatory drugs and gastroprotective agents in at-risk gastrointestinal patients”. Am J Gastroenterol. May; 107(5):707-14.

12. Koffeman AR, Valkhoff VE: "Ischaemic cardiovascular risk and prescription of non-steroidal anti-inflammatory drugs for musculoskeletal complaints." Scand J Prim Health Care.2014 Jun; 32(2):90-8.

13. Sturkenboom MCJM, Burke TA, Tangelder MJD, Dieleman JP, Walton S, Goldstein JL: "Adhrence to proton pump inhibitor or H2-receptor 
antagonists during the use of non-steroidal antiinflammatory drugs." Aliment Pharmacology Ther. 2003 Dec; 18(11-12):1137-47.

14. Van Dijk KN, ter Huurne K, de Vries CS et al. Use of gastroprotective drugs among elderly NSAID users in The Netherlands. Pharm World Sci 2002; 24:100-3.

15. Lanas A, Boers M, Nuevo J. Gastrointestinal events in at-risk patients starting non-steroidal anti-inflammatory drugs (NSAIDs) for rheumatic diseases: the EVIDENCE studyof European routine practice. Ann Rheum Dis. 2013 Dec 18. doi: 10.1136/annrheumdis-2013-204155.

16. Langman MJ, Kahler KH, Kong SX et al. Use of gastroprotective agents and other healthcare resources among patients on nonsteroidal antiinflammatory drugs in the United Kingdom [abstract]. Ann Rheum Dis 1999; 58(Suppl.):202.

17. Schnitzer TJ, Kong SX, Mavros P, Straus WL, Watson DJ. Use of nonsteroidal anti-inflammatory drugs and gastroprotective agents before the advent of cyclooxygenase-2-selective inhibitors: analysis of a large United States claims database. Clin Ther 2001; 23:1984-98.

18. Hogan DB, Campbell NR, Crutcher R, Jennett P, MacLeod N. Prescription of nonsteroidal antiinflammatory drugs for elderly people in Alberta. CMAJ 1994; 151:315-22.

19. Rahme E, Joseph L, Kong SX, Watson DJ, LeLorier J. Cost of prescribed NSAID-related gastrointestinal adverse events in elderly patients. Br J Clin Pharmacol 2001; 52:185-92.

20. Cohen Y, Freeman NL, Kong SX, Arbel D. Gastro intestinal health care resource use and costs associated with nonsteroidal antiinflammatory drugs: analysis of a population cohort in Israel [abstract]. European League Against Rheumatism 2001, Prague, Czech Republic, June 13-16, 2001.
21. Clinard F, Bardou M, Sgro C et al. Non-steroidal anti-inflammatory and cytoprotective drug co-prescription in general practice. A general practitioner-based survey in France. Eur J Clin Pharmacol 2001; 57:737-43.

22. Wolfe F, Anderson J, Burke TA, Arguelles LM, Pettitt D. Gastroprotective therapy and risk of gastrointestinal ulcers: risk reduction by COX-2 therapy. J Rheumatol 2002; 29:467-73. 